\title{
Observation de pentaquarks par l'expérience LHCb
}

Patrick Robbe (robbe@lal.in2p3.fr)

Laboratoire de l'accélérateur linéaire, UMR8607, Université Paris-Sud, BP 34, 91405 Orsay Cedex

\section{L'expérience $\mathrm{LHCb}$ a mis}

en évidence l'existence

d'une nouvelle particule

en analysant l'ensemble

des données enregistrées

lors des collisions au LHC

en 2011 et 2012.

Cette particule a l'originalité

d'être formée de cinq quarks,

dont deux quarks charmés.

Même si cette combinaison

n'est pas interdite par

la théorie des interactions

fortes, c'est la première fois

qu'elle est observée

expérimentalement de façon

non ambiguë.

Plusieurs interprétations

existent pour décrire

la manière dont sont liés

les quarks entre eux dans

ces pentaquarks et prédisent

l'existence d'autres particules

similaires.

\section{L'interaction forte}

Il existe quatre forces fondamentales dans la nature : les forces gravitationnelle, électromagnétique, nucléaire faible et nucléaire forte. C'est cette dernière qui explique que les quarks, particules élémentaires de la matière (dont la liste est donnée dans le tableau 1), sont confinés dans des particules composites appelées hadrons. En effet, cette interaction est si forte que les quarks ne peuvent se trouver isolés. La charge associée à l'interaction nucléaire forte est appelée " couleur » et peut prendre trois valeurs pour un quark, nommées conventionnellement " bleu ", " vert " ou " rouge ", et trois valeurs pour un antiquark, la particule d'antimatière associée au quark: « anti-bleu ", " anti-vert " ou " anti-rouge ". Le modèle des quarks [1] du Modèle Standard de la physique des particules explique leur confinement par le postulat que seuls des états liés incolores peuvent exister. Ainsi, les états liés d'un quark et d'un antiquark (appelés mésons) ou les états liés de trois quarks (appelés baryons, tels le proton ou le neutron) existent, alors que des états liés de deux quarks n'existent pas. Par exemple, une association d'un quark " rouge " et d'un antiquark " anti-rouge " est incolore, alors que celle de deux quarks " rouges " ne le serait pas. De la même façon, un baryon formé d'un quark « rouge », d'un quark "bleu » et d'un quark "vert " est incolore, l'analogie avec la couleur ayant été utilisée pour illustrer ce principe.

L'existence d'états liés contenant plus de quarks que dans ces combinaisons minimales à deux ou trois quarks (mésons et baryons) est prévue par ce modèle, puisque des combinaisons telles que deux quarks et deux antiquarks (" tétraquarks ») ou quatre quarks et un antiquark (" pentaquark ») sont également incolores. Cependant, aucune preuve expérimentale confirmée n'existait jusqu'à récemment.

\section{Les états exotiques}

Les calculs de QCD (Quantum Chromodynamics, ou théorie des interactions fortes) permettent de prédire de façon exacte les spectres de masse des états liés de quarks, que sont les mésons et baryons.

Cependant, des particules ont été observées expérimentalement, dont la masse n'apparaît pas dans ces spectres prédits par la théorie, et sont appelées " états exotiques ". Les plus récents et les plus spectaculaires proviennent du spectre des états liés cic formés d'un quark charmé (c) et d'un antiquark charmé $(\overline{\mathrm{c}})$, qui sont des quarks lourds. (Les particules conventionnelles de ce spectre, prévues par la

\begin{tabular}{|c|c|c|c|c|}
\hline Quark & $\begin{array}{c}\text { Masse } \\
\left(\mathrm{GeV} / \mathrm{c}^{2}\right)\end{array}$ & $\begin{array}{c}\text { Charge } \\
\text { électrique }\end{array}$ & Antiquark & $\begin{array}{c}\text { Charge } \\
\text { électrique }\end{array}$ \\
\hline u (up) & 0,002 & $+2 / 3$ & $\overline{\mathrm{u}}$ & $-2 / 3$ \\
\hline $\mathrm{d}$ (down) & 0,005 & $-1 / 3$ & $\overline{\mathrm{d}}$ & $+1 / 3$ \\
\hline c (charmé) & 1,3 & $+2 / 3$ & $\overline{\mathrm{c}}$ & $-2 / 3$ \\
\hline s (étrange) & 0,1 & $-1 / 3$ & $\overline{\mathrm{s}}$ & $+1 / 3$ \\
\hline $\mathrm{t}$ (top) & 173 & $+2 / 3$ & $\overline{\mathrm{A}}$ & $-2 / 3$ \\
\hline $\mathrm{b}$ (beau ou bottom) & 4,2 & $-1 / 3$ & $\overline{\mathrm{b}}$ & $+1 / 3$ \\
\hline
\end{tabular}

Tableau 1. Liste des quarks et antiquarks. Un quark et son antiquark (par exemple u et ū) ont la même masse . 


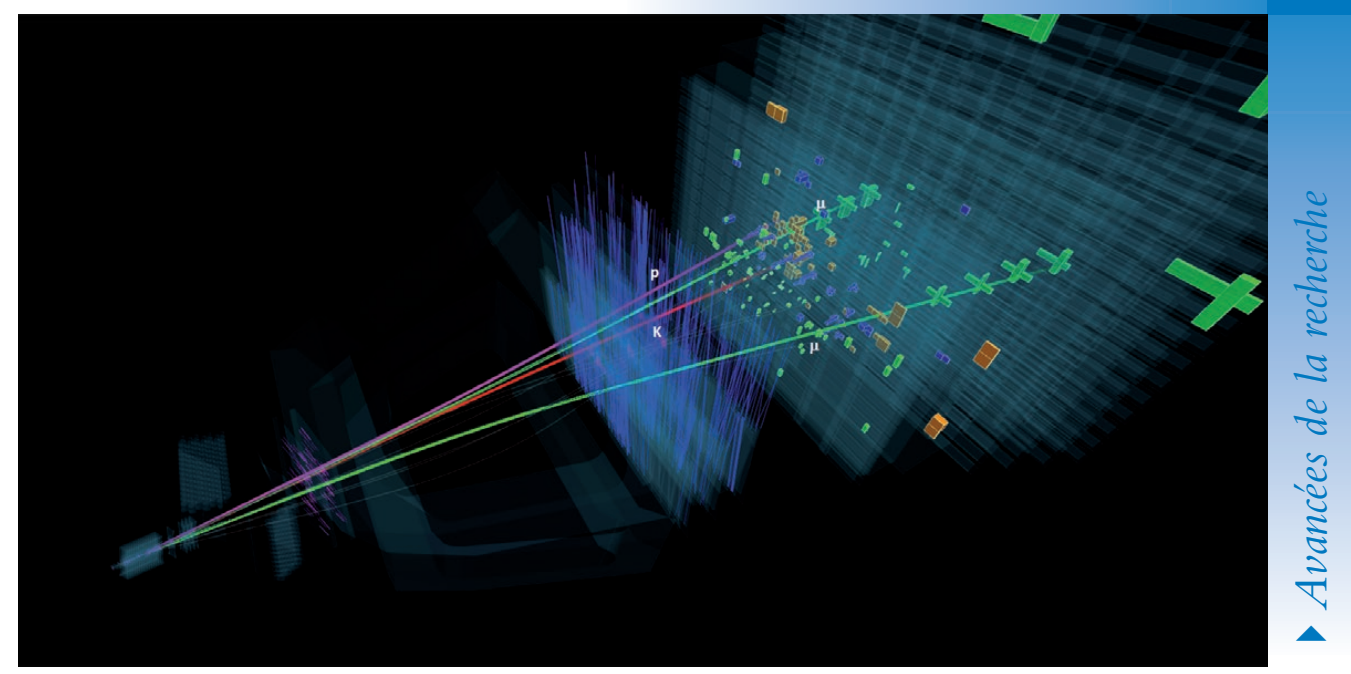

QCD, sont connues avec une bonne précision, la première d'entre elles, le $\mathrm{J} / \psi$, ayant été découverte en 1974.) Ces particules exotiques dans le spectre $c \bar{c}$ ont été d'abord observées au début des années 2000 [2] dans des expériences à relativement basse énergie (de l'ordre de $10 \mathrm{GeV}$ ), BABAR (aux USA), BELLE (au Japon) et BES (en Chine), enregistrant des collisions d'électrons et de positons, mais également dans des expériences à très grande énergie (de l'ordre de $1 \mathrm{TeV}$ ), dans des collisions de protons ou d'antiprotons (Tevatron aux USA et LHC à Genève). À présent, une dizaine de ces états exotiques cc̄ ont été découverts, dont une grande partie par différentes expériences de façon indépendante, ne laissant donc aucun doute quant à leur existence. L'hypothèse que ces états soient des " tétraquarks ", des états liés de deux quarks et deux antiquarks, est une de celles les plus souvent avancées pour décrire ces particules. La confrontation des résultats des différentes expériences, ainsi que l'amélioration de la précision des mesures des caractéristiques de ces particules, permettront de tirer des conclusions définitives.

\section{L'expérience LHCb}

L'expérience $\mathrm{LHCb}$ est un détecteur de physique des particules situé dans un des points d'interaction du LHC, au CERN [3]. Cette expérience est spécialisée dans l'étude des mésons et baryons formés des quarks c et aussi des quarks lourds b ("beaux »), qui sont créés lors des collisions du LHC. Pendant la première campagne de fonctionnement de l'accélérateur, entre 2010 et 2012, $210^{12}$ particules contenant un quark b ont été produites dans l'expérience, et c'est parmi leurs désintégrations qu'ont été découverts des pentaquarks. La figure 1 montre un événement contenant un pentaquark, enregistré en 2012 par l'expérience.

1. Reconstruction d'un événement contenant un candidat pentaquark dans le détecteur LHCb.

Les trajectoires reconstruites des particules produites dans la désintégration du pentaquark, $P_{c} \rightarrow J / \psi p$, sont montrées : en vert pour les deux muons de la désintégration du $\mathrm{J} / \psi \rightarrow \mu^{+} \mu^{-}$, en violet pour le proton $p$. Ce pentaquark provient de la désintégration d'un $\Lambda_{b} \rightarrow P_{c} K^{-}$, la trajectoire du $K^{\top}$ est représentée en rouge. Les particules $p, \mu^{+}$et $\mu^{-}$proviennent potentiellement de la désintégration d'un pentaquark, car l'événement a été sélectionné parmi ceux pour lesquels la masse invariante calculée pour le $\Lambda_{b}$ se situe dans le pic visible sur la figure 2, et la masse invariante calculée pour le $P_{c}$ dans le pic visible sur la figure 3.

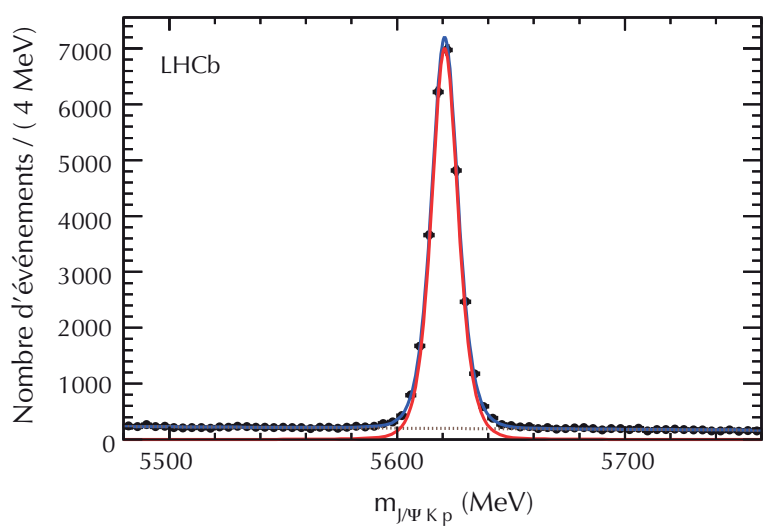

2. Distribution de la masse $\Lambda_{\mathbf{b}}{ }^{0}$ (nombre de recombinaisons reconstruites par intervalles de $4 \mathrm{MeV}$ ).

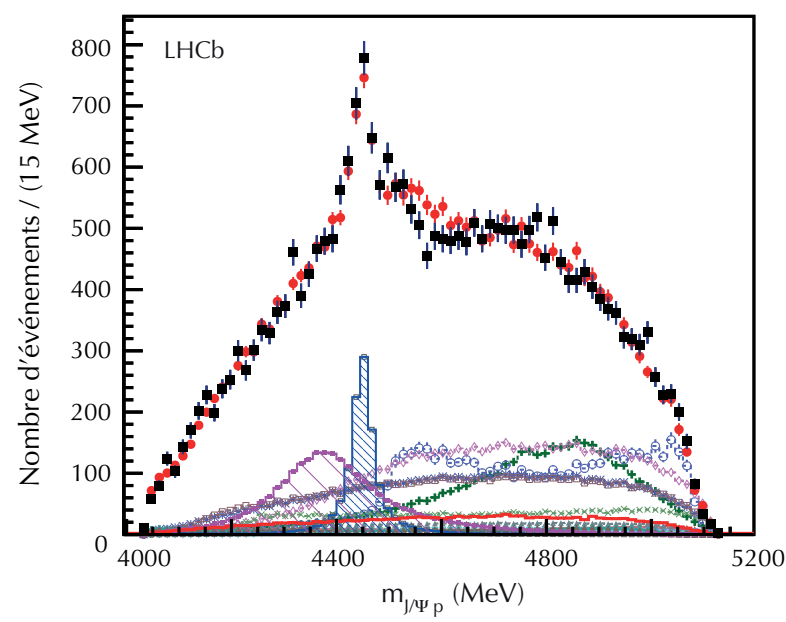

3. Distribution de masse invariante de la combinaison $\mathrm{J} / \psi \mathbf{p}$ (nombre de combinaisons reconstruites par intervalles de $15 \mathrm{MeV}$ ). La contribution du pentaquark de masse $4450 \mathrm{MeV}$ est montrée par l'histogramme bleu, celle du pentaquark de masse $4380 \mathrm{MeV}$ par l'histogramme violet. Les autres combinaisons qui contribuent à cette distribution proviennent de désintégrations non résonantes dans la chaîne de désintégration J/ $\psi$ p K', ou bien de résonances connues $\Lambda^{*} \rightarrow p K$, dont les réflexions sont indiquées par les autres histogrammes. 
$>>$

\section{La découverte des pentaquarks}

Les états pentaquarks ont été découverts par hasard, au cours d'une analyse pour mesurer le temps de vie de la particule $\Lambda_{\mathrm{b}}$ [4]. Cette particule est un baryon contenant un quark b et deux quarks légers, $u$ et $d$, et de temps de vie de l'ordre de 1,5 ps. Elle se désintègre par interaction faible dans un grand nombre de modes différents, dont le mode de désintégration $\Lambda_{\mathrm{b}}{ }^{0} \rightarrow \mathrm{J} / \psi \mathrm{K}^{-} \mathrm{p}$, utilisé dans cette analyse. Le proton $\mathrm{p}$ et le kaon $\mathrm{K}^{-}$(méson formé par un quark s et un antiquark $\overline{\mathrm{u}}$ ) sont détectés directement dans le détecteur, et la particule $\mathrm{J} / \psi$ (méson formé d'un quark c et d'un antiquark c) est détectée dans le mode de désintégration $\mathrm{J} / \psi \rightarrow \mu^{+} \mu^{-}$(un muon et un antimuon). Un très grand nombre de désintégrations (26 000) sont ainsi détectées avec très peu de bruit de fond, comme le montre la figure 2 , qui est le spectre de masse invariante des combinaisons $\mathrm{J} / \psi \mathrm{K}^{-}$p. La masse invariante est calculée à partir des masses et des impulsions des produits de désintégration, mesurées dans le détecteur. Pour des combinaisons provenant de la désintégration de vrais $\Lambda_{b}{ }^{0}$, cette masse est proche de la masse connue du $\Lambda_{\mathrm{b}}{ }^{0}$ (environ $5620 \mathrm{MeV}$ ), alors que pour des combinaisons aléatoires ne provenant pas de cette désintégration, sa valeur est arbitraire. Ceci explique le pic visible sur la figure 2 autour de la masse du $\Lambda_{b}{ }^{0}$, contenant les $\Lambda_{\mathrm{b}}{ }^{0}$ reconstruits dans l'expérience, et indiqué par la courbe rouge. $\mathrm{La}$ forme de ce pic est due à la précision de la mesure des impulsions par le détecteur, qui n'est pas parfaite et introduit des différences entre la mesure expérimentale et la vraie masse de la particule.

Cette désintégration avec trois particules dans l'état final peut se produire par l'intermédiaire d'une autre particule, de temps de vie très court, appelée résonance et notée $\mathrm{P}_{\mathrm{c}}$ dans ce cas, c'est-à-dire avec une désintégration en deux corps, suivie par une autre désintégration en deux corps de cette résonance pour obtenir l'état final. Dans le cas présent, il s'agit de $\Lambda_{\mathrm{b}} \rightarrow \mathrm{P}_{\mathrm{c}} \mathrm{K}^{-}$, suivie de la désintégration $\mathrm{P}_{\mathrm{c}} \rightarrow \mathrm{J} / \psi \mathrm{p}$. Ceci est généralement mis en évidence en regardant le spectre en masse invariante de ces deux particules, $\mathrm{J} / \psi$ et $\mathrm{p}$. La figure 3 représente ce spectre mesuré par LHCb, les carrés noirs montrant la distribution observée dans les données. On y remarque un pic inattendu.
Des vérifications détaillées des données ont permis d'exclure que ce pic soit dû à des réflexions d'autres résonances connues, ou à des erreurs d'identification des particules de l'état final. Ces observations permettent de conclure qu'il s'agit bien d'une nouvelle particule, de masse $4380 \pm 30 \mathrm{MeV}$, de largeur de résonance $205 \pm 88 \mathrm{MeV}$, de spin 3/2 et de parité négative. Les données montrent aussi la présence d'une seconde résonance, avec une masse de $4450 \pm 3 \mathrm{MeV}$ et une largeur plus petite, de $39 \pm 20 \mathrm{MeV}$. Ces deux résonances correspondent à deux états d'une nouvelle particule qui a été nommée $\mathrm{P}_{\mathrm{c}}^{+}$. Les cercles rouges sur la figure 3 montrent l'ajustement des données par le modèle à deux résonances.

\section{Les différentes interprétations et perspectives}

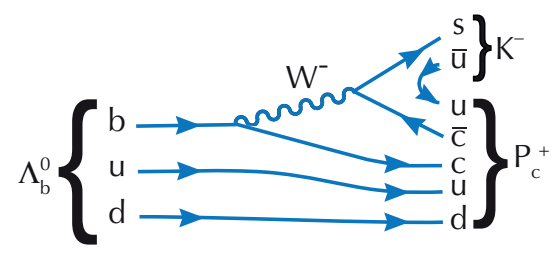

4. Diagramme de la désintégration $\Lambda_{\mathrm{b}}{ }^{0} \rightarrow \mathrm{P}_{\mathrm{c}}^{+} \mathrm{K}^{-}$.

Le diagramme en quarks de la désintégration du $\Lambda_{\mathrm{b}}$ est donné dans la figure 4. Le $\mathrm{P}_{\mathrm{c}}{ }^{+}$ se désintègre en $\mathrm{J} / \psi \mathrm{p}$, par interaction forte. Puisque le $\mathrm{J} / \psi$ est un état lié $c \overline{\mathrm{c}}$, le $\mathrm{P}_{\mathrm{c}}{ }^{+}$ doit contenir aussi ces deux quarks; le proton $\mathrm{p}$ de l'état final étant un baryon, le $\mathrm{P}_{\mathrm{c}}{ }^{+}$doit contenir au moins ses trois quarks. Le contenu minimum en quarks du $\mathrm{P}_{\mathrm{c}}^{+}$est donc c̄̄ uud, c'est-à-dire cinq quarks. Ces quarks peuvent être liés entre eux de façon forte et confinés dans un volume unique. Mais ils pourraient aussi former des «molécules » associant deux particules traditionnelles (un baryon et un méson) faiblement liées. La figure 5 illustre schématiquement ces deux possibilités.

D'autres expériences au LHC (ATLAS et CMS) enregistrent également des données en même temps que $\mathrm{LHCb}$ et pourraient aussi observer ces pentaquarks et améliorer la précision sur la mesure de leurs masses. Pour pouvoir en apprendre plus sur les pentaquarks, il faut en découvrir d'autres qui se désintègrent de manière différente ; la construction expérimentale de leur spectre en masse, de leur largeur et de leurs propriétés intrinsèques permettront d'apprendre comment ils sont formés.
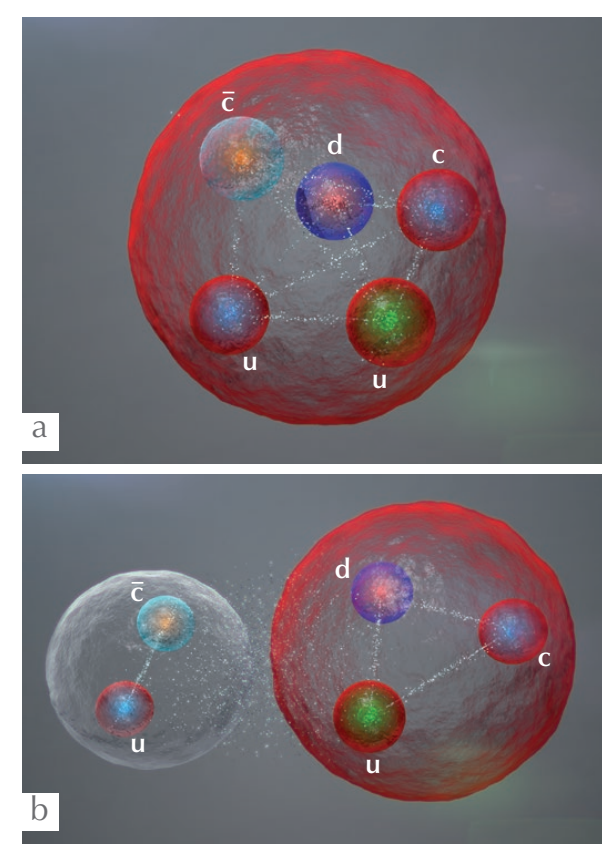

5. Illustration d'un pentaquark formé de cinq quarks liés fortement (a) ou d'une molécule formée d'un méson et d'un baryon contenant aussi au total cinq quarks (b).

Jusqu'à présent, ces pentaquarks ont été observés dans un mode de désintégration d'un hadron contenant un quark b, mais ils pourraient aussi être produits de façon directe ; si c'était le cas, cela permettrait de distinguer parmi les hypothèses théoriques la nature exacte de ces particules.

Le relevé de données au LHC a redémarré en 2015, et une grande quantité de collisions ont été déjà enregistrées. Leur analyse devrait permettre d'apprendre beaucoup plus sur les pentaquarks et sur le mécanisme exact qui lie ces quarks entre eux.

\section{Références}

1- M. Gell-Mann, "A schematic model of baryons and mesons", Phys. Lett. 8 (1964) 214.

2• S.-K. Choi et al. (BELLE Collaboration), “Observation of a narrow charmoniumlike state in exclusive $\mathrm{B}^{ \pm} \rightarrow \mathrm{K}^{ \pm} \pi^{+} \pi^{-} \mathrm{J} / \psi$ decays", Phys. Rev. Lett. 91 (2003) 262001.

3• F. Machefert, « Recherche d'une physique au-delà du modèle standard et étude de la violation de CP avec l'expérience LHCb au CERN », Reflets de la physique 43 (2015) 18.

4- R. Aaij et al. (LHCb collaboration), "Observation of $\mathrm{J} / \psi \mathrm{p}$ resonances consistent with pentaquark states in $\Lambda_{b}{ }^{0} \rightarrow \mathrm{J} / \psi \mathrm{K}^{-} \mathrm{p}$ decays", Phys. Rev. Lett. 155 (2015) 072001. 